\title{
Necessidades pedagógicas sob a ótica da supervisão de estágio curricular em terapia ocupacional
}

\section{Pedagogical needs from the perspective of curriculum internship supervision in occupational therapy}

\author{
Adriana Reis de Barros ${ }^{1}$ (D) $\mid$ adriana.barros@uncisal.edu.br \\ Rozangela Maria de Almeida Fernandes Wyszomirska² (D) rozangela.wyszomirska@famed.ufal.br \\ Kerle Dayana Tavares de Lucena' (D) krele.lucena@uncisal.edu.br
}

\section{RESUMO}

Introdução: O estágio curricular é um momento de integração dos discentes com o mundo do trabalho, etapa indispensável no processo de desenvolvimento e aprendizado de competências específicas da atividade profissional, de acordo com os princípios e as diretrizes do Sistema Único de Saúde.

Objetivo: Este estudo teve como objetivo compreender as necessidades pedagógicas dos supervisores do estágio supervisionado do curso de Terapia Ocupacional e as características desejáveis em um supervisor de estágio, a partir da visão dos discentes.

Método: Trata-se de um estudo transversal e exploratório de natureza qualitativa, com uso de análise temática de conteúdo, compreendida em três etapas: pré-análise, exploração de material e tratamento dos resultados. Participaram do estudo 24 supervisores de estágio e 15 discentes do curso de Terapia Ocupacional que preencheram dois formulários semiestruturados on-line: um para os supervisores e outro para os alunos.

Resultado: Para os supervisores, a presença do aluno no serviço contribui para as trocas no cotidiano do trabalho e instiga o preceptor a buscar mais conhecimento, porém esperam maior reconhecimento da atuação do supervisor e consideram importante um fluxo contínuo para a formação em docência dos profissionais do serviço, em especial sobre avaliação de desempenho e práticas inovadoras. Por sua vez, os discentes têm a expectativa de encontrar supervisores que apresentem, de forma segura, contribuições significativas para a aprendizagem por meio da experiência profissional.

Conclusão: Este estudo possibilitou compreender as necessidades pedagógicas da supervisão de estágio, a relevância da formação para docência nos serviços e a importância do acolhimento e acompanhamento dos discentes com orientações ao longo do processo de aprendizado. A partir desses pressupostos, foi desenvolvido um desenho de curso para capacitação pedagógica na modalidade a distância para supervisores de estágio.

Palavras-chave: Estágio Supervisionado; Profissional de Saúde; Ensino; Educação Permanente.

\section{ABSTRACT}

Introduction: Curricular internship supervised by professors and professionals from services linked to the Unified Health System.

Objective: To understand the pedagogical needs of internship supervisors in the occupational therapy course and the desirable characteristics of an internship supervisor, from the student perspective.

Method:Cross-sectional and exploratory study of a qualitative nature, using thematic content analysis, comprising three stages: pre-analysis, exploration of material with treatment of results. Twenty-four internship supervisors and fifteen students from the occupational therapy course participated in the study, using two online semi-structured forms, one for supervisors and one for students.

Results and Discussion: For the supervisors, the presence of the student in the service contributes to exchanges in the daily work, and instigates the preceptor to seek more knowledge, but they expect greater recognition of the supervisor's performance and considered it important to have a continuous flow for training in teaching service professionals, in particular on performance evaluation and innovative practices. Students expect to find supervisors who make significant contributions to their learning in a safe way, through professional experience.

Conclusion: The study revealed the pedagogical needs of internship supervision, the importance of training for teaching in services, and the importance of welcoming and monitoring students with guidance throughout the learning process. Based on these assumptions, a course design was developed for remote pedagogical training for internship supervisors

Keywords: Supervised Internship; Healthcare Professional; Teaching; Permanent Education.

${ }^{1}$ Universidade Estadual de Ciências da Saúde de Alagoas, Maceió, Alagoas, Brasil.

${ }^{2}$ Universidade Federal de Alagoas, Maceió, Alagoas, Brasil.

Editora-chefe: Rosiane Viana Zuza Diniz.

Editora associada: Izabel Cristina Meister Martins Coelho.

Recebido em 21/11/21; Aceito em 06/01/22.

Avaliado pelo processo de double blind review. 


\section{INTRODUÇÃO}

O estágio curricular é um momento de integração dos discentes com o mundo do trabalho, etapa indispensável no processo de desenvolvimento e aprendizado de competências específicas da atividade profissional, de acordo com os princípios e as diretrizes do Sistema Único de Saúde (SUS) ${ }^{1,2}$. Esse tipo de estágio foi regulado pela Lei no $11.788 / 2008$, sendo caracterizado como um ato educativo supervisionado e desenvolvido em ambiente de trabalho ${ }^{3}$.

O estágio é uma atividade curricular que permite ao discente promover a articulação entre os conhecimentos construídos na academia e as demandas dos serviços. Favorece o crescimento pessoal mediante o desenvolvimento de ações vivenciadas que permitam experiências significativas e motivadoras, para que os alunos possam adquirir uma identidade profissional na sua atuação ${ }^{4-6}$.

Devido à complexidade, o processo de ensino das práticas deve ser desenvolvido de forma articulada entre o centro formador e os serviços de saúde, sempre alinhados aos princípios do SUS?

Nesse sentido, uma boa articulação entre a universidade e os serviços consiste em uma ferramenta fundamental para a integração entre teoria e prática. Pimentel et al..$^{8}$ apontam a importância em ampliar discussões acerca da realidade dos serviços em busca de soluções para os problemas do cotidiano das práticas.

\section{O supervisor e profissional nos serviços da saúde}

O ensino das práticas em saúde é um processo singular que envolve a inserção do aprendiz nos espaços de assistência à comunidade em geral. Nesse contexto, o cenário de aprendizagem é privilegiado pela produção do conhecimento com a experiência na prática, além da construção de relações, o que é fundamental para o desenvolvimento profissional ${ }^{9}$.

A partir das vivências que possibilitam a inserção dos estudantes na realidade dos serviços, tal qual ela se apresenta, o profissional de saúde que os recebe tem a função de contribuir para a formação de indivíduos críticos e reflexivos diante das exigências da futura prática profissional ${ }^{10-12}$.

Nesse contexto, o supervisor ou preceptor é um trabalhador do serviço e tem responsabilidades com assistência, além de exercer funções de ensinar, acompanhar, orientar e inserir o estudante nas equipes dos serviços². O supervisor é um profissional que deve apresentar conhecimento teórico e didático, cuja experiência e discernimento são fundamentais para interligar a universidade e o serviço a fim de oferecer habilidades específicas no que concerne à humanização e ética, atuando como orientador e incentivador da aprendizagem ${ }^{13-15}$.
Cerqueira ${ }^{16}$ e Martins ${ }^{17}$ compreendem o preceptor como um educador e ressaltam a importância do seu papel no contexto de encontros entre estudantes usuários e outros profissionais como uma etapa importante para a formação em saúde. Nesse contexto, o cenário de aprendizagem é caracterizado não somente pelo aprender-conhecer e pelo aprender-fazer, mas também pelo aprender-ser e pelo aprender-conviver, ou seja, é um cenário de construção de relações. Nessa perspectiva, todos são beneficiados porque essa aprendizagem é estruturada em torno de vivências em cenários permeados pelas necessidades de saúde das pessoas ${ }^{9,18,19}$.

Tendo em vista a complexidade que abrange o ensino nos cenários de prática, Pimentel et al. ${ }^{8}$ fazem referência a uma função mediadora que demanda disponibilidade para orientação, facilitação, aconselhamento e escuta dos alunos. Soares et al. ${ }^{20}$ e Oliveira ${ }^{18}$ fazem menção à função do profissional de ensino nos campos de prática como um ator em ato, que trabalha nas cenas da vida real, proporcionando aprendizado a partir das vivências no cotidiano dos serviços.

O perfil e as atividades do preceptor devem ser pactuados previamente nos programas dos cursos. O conhecimento de metodologias de ensino e aprendizagem e a aplicação delas, além da discussão sobre a importância de métodos de avaliação da prática profissional no local de trabalho, são fatores fundamentais para a educação no trabalho em saúde ${ }^{21,22}$.

\section{As práticas pedagógicas no processo de estágio supervisionado}

Grande parte dos profissionais de saúde não tem formação para o ensino. Encontramos em Mohr ${ }^{23}$ uma boa reflexão ao retratar que a maioria dos currículos da graduação dos cursos da área da saúde não apresenta conhecimentos específicos como os pedagógicos, muitas vezes presentes nas suas práticas profissionais, como planejar e executar ações educativas de cuidado com os usuários e familiares.

Alguns estudos apontam como desafio para o exercício da preceptoria e relacionados aos macroproblemas no âmbito da preceptoria no SUS o despreparo pedagógico para avaliar, desenvolver pesquisas e planejar atividades com o uso de tecnologias educacionais que potencializam a aprendizagem, além da baixa valorização das atividades de preceptoria ${ }^{1,24-26}$.

Sobre essas afirmativas, Lacerda et al. ${ }^{12}$ trazem a importância de investimentos nos processos de ensino nos serviços para a formação de novos profissionais e afirmam que a maioria dos preceptores atua empiricamente sem uma formação específica para desenvolver um processo de ensinoaprendizagem.

Ainda sobre formação, Girotto ${ }^{27}$ refere que quanto mais bem preparado for o profissional que recebe o estudante 
na prática, maior será a chance de alcançar os objetivos educacionais para o ensino de futuros profissionais. De fato, os preceptores que participam de iniciativas educacionais estão mais bem preparados para enfrentar os desafios e são mais satisfeitos com a função de preceptor ${ }^{28}$.

Diante do exposto, a proposta do presente estudo foi compreender as principais necessidades pedagógicas do processo de supervisão de estágio, bem como as principais características dos supervisores, na visão do discente do curso de Terapia Ocupacional de uma universidade pública.

\section{MÉTODO}

Trata-se de um estudo transversal e exploratório de natureza qualitativa, em que se utilizou a análise temática do conteúdo que, de acordo com Bardin ${ }^{29}$, funciona por operações de desmembramento do texto em unidades e em categorias, segundo reagrupamento analógico.

\section{Coleta dos dados}

A coleta dos dados ocorreu de 23 de abril a 20 de junho de 2020.

Fizeram parte do estudo 24 supervisores de estágio do curso de Terapia Ocupacional de uma universidade pública de Alagoas, incluindo 12 profissionais do serviço e 12 docentes do curso que exercem função de supervisão no campo das práticas, e 15 discentes matriculados no Estágio Supervisionado Obrigatório (ESO).

Como instrumento de coleta de dados, utilizaram-se dois formulários semiestruturados, com perguntas fechadas e abertas: um (com 15 perguntas) para os supervisores e outro (com quatro perguntas) para os discentes regularmente matriculados no curso. Disponibilizaram-se os dois formulários na plataforma Google Forms, os quais foram confeccionados pela pesquisadora.

Os procedimentos utilizados para a coleta e identificação dos dados ocorreram em cinco etapas: 1. levantamento do quantitativo de supervisores de estágio e discentes com devido contato de e-mail e telefone, por meio da coordenação do curso e da coordenação de estágio; 2. utilização de uma simulação teste para aplicação do formulário on-line; 3. envio de convite com esclarecimentos sobre a proposta do estudo por aplicativo de mensagem WhatsApp para os supervisores e discentes; 4. envio do Termo de Consentimento Livre e Esclarecido (TCLE) como requisito obrigatório para o link de acesso ao formulário no Google Forms, para participação da pesquisa via aplicativo de mensagem WhatsApp e endereço eletrônico (e-mail) de alguns participantes que assim solicitaram; 5 . armazenamento da produção dos dados coletados.

\section{Análise dos dados}

A análise de conteúdo organiza-se em torno de três polos cronológicos $^{31}$ descritos como: 1. pré-análise; 2. exploração do material; e 3. tratamento dos resultados.

A pré-análise (Figura 1) foi a etapa de organização para sistematizar e operacionalizar as ideias com uma leitura flutuante e aprofundada do texto na busca dos objetivos: compreender as principais necessidades pedagógicas dos supervisores de estágio do curso de Terapia Ocupacional; descrever o perfil dos supervisores de estágio do curso de Terapia Ocupacional de uma universidade pública; identificar as principais necessidades pedagógicas dos preceptores nas práticas de estágio; conhecer as características desejáveis do supervisor de estágio, a partir da visão dos discentes, sobre o acesso e acolhimento nos serviços e, ainda nessa fase, com a predeterminação das categorias temática de categorização e definição das regras de codificação das unidades de referência (UR) e de contexto (UC).

A etapa de exploração do material (Figura 2) consistiu na operação de codificação, transformação por recorte, agregação e decomposição em UR, UC e temas.

De acordo com Bardin ${ }^{29}$, essa fase não se limita ao conteúdo, embora a autora afirme o seguinte: "Codificação corresponde a uma transformação dos dados brutos do texto,

Figura 1. Fase da pré-análise.

\section{FASE I - PRÉ-ANÁLISE}

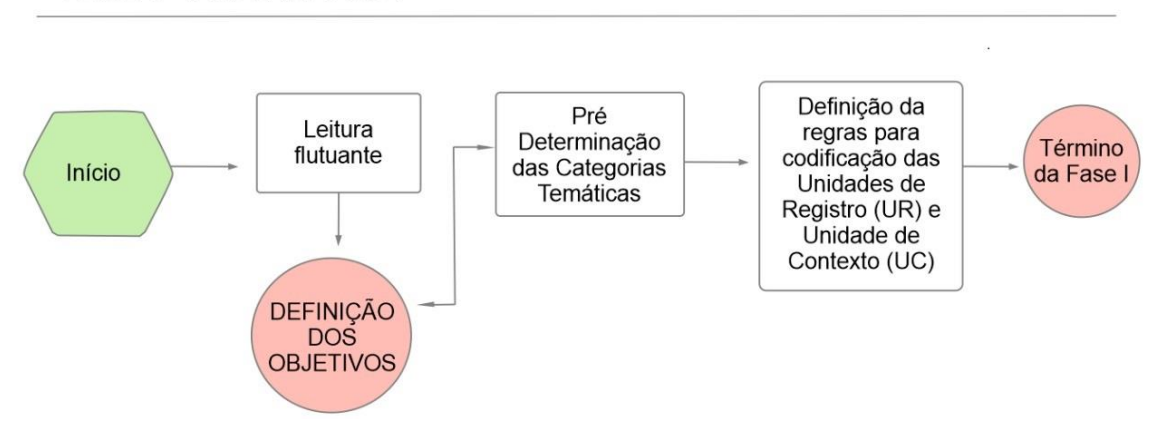

Fonte: Elaborada pelas autoras. 
transformação com regras precisas, por recortes, agregação e enumeração, permite atingir uma representação do conteúdo ou da sua expressão".

O tratamento dos resultados (Figura 3) culminou com a identificação de duas grandes categorias - supervisão acadêmica como ferramenta potente de educação transformadora e abordagens pedagógicas nos espaços de aprendizagem do ensino-serviço - e de duas subcategorias (Quadro 1) a partir das evidências encontradas na construção da discussão do trabalho, bem como na elaboração de estratégias de educação permanente e multimídia, que possam ser reaplicadas em outros cursos, ampliando o alcance para outras áreas na formação em saúde.

\section{Aspectos éticos}

O projeto foi aprovado pelo Comitê de Ética em Pesquisa da Universidade Estadual de Ciências da Saúde de Alagoas
(Uncisal) em abril de 2020: Certificado de Apresentação para Apreciação Ética (CAAE) n 29749020.4.0000.5011.

\section{RESULTADOS E DISCUSSÃO}

Com base nos dados elencados, apresentam-se a seguir as categorias identificadas por meio da análise de temática de conteúdo, que foi empregada por permitir acessar os núcleos dos sentidos que compõem uma comunicação.

A partir do material de pesquisa, foi possível estabelecer a criação de duas categorias temáticas e duas subcategorias com base no referencial teórico apresentado (Quadro 1).

\section{Supervisão acadêmica como ferramenta potente de educação transformadora.}

Para essa categoria, identificaram-se as duas subcategorias apresentadas a seguir.

Figura 2. Fase da exploração do material.

\section{FASE II - EXPLORAÇÃO DO MATERIAL}

Início

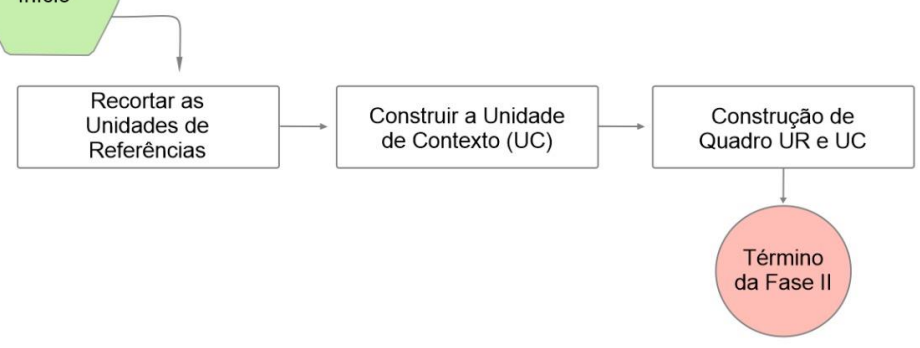

Fonte: Elaborada pelas autoras.

Figura 3. Fase de tratamento dos resultados.

FASE III - TRATAMENTO DOS RESULTADOS, A INFERÊNCIA E A INTERPRETAÇÃO

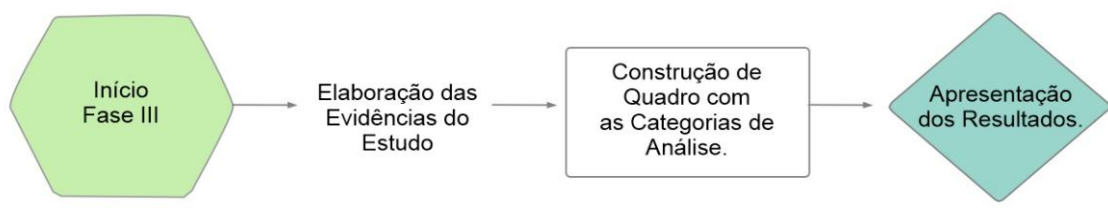

Fonte: Elaborada pelas autoras.

Quadro 1. Categorias e subcategorias temáticas de análise.

\section{Categoria temática de análise}

1 - Supervisão acadêmica como ferramenta potente de educação transformadora

2 - Abordagens pedagógicas nos espaços de aprendizagem do ensino-serviço

\section{Subcategoria}

A - A função do supervisor como educador e a presença do estudante nos serviços

B - O que o discente espera de um bom supervisor no campo de estágio

C - Diálogo entre ensino e serviço

D - Formação para docência

Fonte: Elaborado pelas autoras. 


\section{A função do supervisor como educador e a presença do estudante nos serviços}

Essa subcategoria trata sobre a presença do estudante que é inserido no cotidiano dos serviços e participa da dinâmica dos atendimentos, de modo a oportunizar a troca de saberes no cotidiano do trabalho, os saberes do discente e a experiência dos profissionais nos cenários de prática.

A presença do aluno traz motivação para o serviço [...] proporcionando atualização e dinamismo aos processos de trabalho e aos atendimentos (P14).

A presença deles é motivadora, me estimula a estar sempre atualizado (P12).

O preceptor conduz o processo e transforma as atividades desenvolvidas no âmbito do trabalho em momentos educacionais, que participam como elemento de mediação entre dois polos de atuação: o mundo da teoria e o da prática ${ }^{22,31}$.

Percebe-se ainda a partir dos relatos a troca de experiência entre os supervisores e discentes, com ensino e aprendizagem no campo da prática profissional, nos diversos cenários de estágio.

Aprendizado constante e uma troca recíproca (P13).

A presença do aluno contribui com as práticas no serviço e traz atualidades acadêmicas e inova as atividades oferecidas (P22).

A preceptoria como espaço de troca de experiências é bastante positiva e propicia atualização teórica ao preceptor. Quando associada às práticas de ensino no serviço, traz benefícios para os serviços de saúde e as comunidades ${ }^{18}$.

Embora o supervisor apresente-se satisfeito com a presença dos alunos, acredita-se que é necessário um maior reconhecimento da atuação do supervisor com os estudantes e com o ensino nos serviços.

As demandas do estágio somam-se as demandas do serviço (P5).

Contribui em vários aspectos no serviço. Traz atualidades acadêmicas e inova as atividades oferecidas (P21).

De acordo com Correa et al. ${ }^{28}$, a prática pedagógica no ambiente de trabalho, conduzida por profissionais capacitados, mais especificamente no que concerne ao aspecto pedagógico, é importante para alcançar os objetivos da preceptoria.

Quando questionados sobre os conteúdos pedagógicos necessários ao ensino dos estágios, os preceptores apontam a formação e o treinamento como temáticas importantes para melhorar o desempenho da supervisão de estágio.
Curso de formação para o preceptor/docente sobre avaliação de desempenho (P9).

Formação para profissionais não docentes que estão recebendo os estagiários $(P 11)$

Ferreira et al. ${ }^{30}$ identificaram alguns saberes e competências que os preceptores precisam desenvolver, como ser proativo em sua formação, aprender a refletir sobre sua prática e modificá-la quando necessário, desenvolver atividade de pesquisa, como também adquirir conhecimentos didático/ pedagógicos.

O profissional de saúde tem dupla função. Girotto ${ }^{27}$ complementa que saber ensinar exige aprimoramento constante no trabalho desse profissional, que exerce também a função de supervisor, sendo corresponsável pela formação profissional. Silva et al. ${ }^{22}$ destacam a importância de um embasamento teórico para as práticas e de investimentos em métodos de avaliação no local de trabalho.

\section{O que o discente espera de um bom supervisor no campo de estágio}

Sabe-se que as vivências do estágio, como etapa formativa, possibilitam o aprimoramento dos conhecimentos teóricos e práticos, e proporcionam o amadurecimento e a responsabilidade do discente no cotidiano profissional. Com base nos depoimentos dos discentes sobre o que esperam de um bom supervisor nos espaços de prática, identificaram-se os seguintes aspectos:

Que esteja presente e guiando o estagiário para que ele se sinta seguro em atender e fazer o estágio (P7).

Que supervisione e esteja atento às demandas dos estagiários, que dê suporte e prepare para ser profissional (P4)

Quanto mais preparado para o exercício pedagógico do ensino dos estudantes no ambiente de trabalho, maior será o aproveitamento do estágio e mais perto de alcançar os objetivos educacionais ${ }^{27}$.

Em relação às contribuições do supervisor e, sobretudo, no que diz respeito às orientações, os discentes esperam que sejam significativas para promover a aprendizagem, além da experiência de ser inserido nas equipes de trabalho e promover reflexões sobre as potencialidades e fragilidades.

Didático, trabalhar em conjunto (P1).

O supervisor deve apresentar o serviço e ter diálogo aberto [...] (P12).

Que seja uma pessoa disposta a ajudar, que incentivam os estagiários, que pontuem qualidades e falhas (P13). 
O preceptor deve apresentar conhecimento teórico e ser didático, além de responsabilizar-se pela orientação, explicação, escuta, aproximação e inserção do estudante no processo de trabalho e também na equipe multiprofissional com orientações das práticas, de acordo com os planos de ensino ${ }^{14,32}$.

Encontramos em Gubert et al. ${ }^{33}$ um estudo sobre formação dos profissionais e educação nos serviços, no qual as autoras afirmam que é necessária a capacitação docente para o exercício no campo e pontuam que a integração entre docência e assistência agrega qualidade ao processo de formação e evita dicotomia entre teoria e prática. Entre as necessidades de aperfeiçoamento profissional, destacam-se o compromisso com a aprendizagem do aluno, o conhecimento do papel do preceptor como um formador e a capacidade de incentivar o aluno para sua aprendizagem ${ }^{34}$.

Em estudo de revisão de literatura sobre definições do papel de preceptor na saúde, Teixeira et al. ${ }^{35}$ identificaram no preceptor um profissional que serve como elo entre o ensino e o serviço, atuando como um facilitador do processo de aprendizagem do aluno para que este possa formar competências para a prática profissional.

Com base nos estudos dos autores mencionados, foi encontrado um alinhamento com as falas e os desejos dos discentes.

\section{Abordagens pedagógicas nos espaços de aprendizagem do ensino-serviço}

Para essa categoria, identificaram-se as duas subcategorias apresentadas a seguir.

\section{Diálogo entre ensino e serviço}

Nas respostas dos entrevistados, percebem-se a importância de investimento e aproximação na relação entre a universidade e os serviços, e a necessidade de melhorar a articulação entre teoria e prática.

Para melhorar a presença dos acadêmicos nas unidades, deveria haver uma melhor articulação da IES e a Secretaria Municipal de Saúde (P2).

Dialogar com as equipes de saúde sobre a importância dos estágios para formação de futuros profissionais (P1).

Ribeiro et al. $^{2}$ mencionam a necessidade de reduzir o distanciamento entre os preceptores do campo das práticas e as instituições de ensino superior (IES), enquanto Ferreira ${ }^{36}$ destaca a inter-relação entre ensino e serviço como uma estratégia eficaz por permitir a troca entre os serviços de saúde e profissionais com a universidade, de modo a favorecer a qualificação dos serviços e das pesquisas.
Assim, a experiência do supervisor é fundamental para interligar a academia e o trabalho, e os serviços são terreno fértil para o desenvolvimento das habilidades específicas ${ }^{14}$.

Com base nas falas dos supervisores, nota-se uma necessidade de acompanhamento da universidade para aprimorar questões do ensino-aprendizagem, estreitar as relações e otimizar a comunicação entre os envolvidos.

Uma discussão sobre o projeto pedagógico do curso e o planejamento do estágio (P3).

[...] melhorar a integração ensino-serviço (P4).

Dialogar sobre a importância dos estágios para formação, aproximando a teoria da prática (P1).

Junqueira et al. ${ }^{37}$, por meio de estudos sobre ensino na graduação e desenvolvidos nos cenários de prática no SUS, identificaram a importância da articulação entre as instituições formadoras e os serviços.

\section{Formação para docência}

Como subcategoria de análise, identificaram-se, nos relatos dos supervisores, alguns conteúdos relacionados ao processo de ensino-aprendizagem, como a importância da correlação entre teoria e prática, e a avaliação da aprendizagem, aspectos considerados relevantes para a supervisão dos estágios.

No relato dos supervisores, destaca-se o processo de capacitação docente:

É importante atualização no processo de avaliação da aprendizagem (P3).

Formação para avaliação da aprendizagem para a preceptoria, discussão sobre o planejamento do estágio (P5).

Fazer correlação teórico-prática na preceptoria, inserir o estudante no serviço e junto à equipe, e avaliação da aprendizagem (P2).

As estratégias didáticas devem permear as práticas de ensino nos serviços. Ribeiro et al. ${ }^{31}$ acreditam que apenas o conhecimento sobre o conteúdo não é suficiente. É necessário que o supervisor ou preceptor tenha expertise da prática pedagógica.

Observa-se, nas falas dos supervisores, a necessidade de aprimoramento sobre o processo avaliativo da aprendizagem, de correlação entre a teoria e prática, bem como de formação e capacitação em docência para profissionais não docentes.

Capacitação dos profissionais em relação às práticas inovadoras (P3).

Constante treinamento com os preceptores de estágios (P12). 
Apoio no processo ensino-aprendizagem como a avaliação de processo de ensino e aprendizagem (P2).

Concordando com Ribeiro et al. ${ }^{31}$, para ensinar, é necessário conhecimento para além do conteúdo da disciplina. Considerando que a preceptoria é uma prática de educação no trabalho, o preceptor precisa de formação pedagógica para receber o estudante no local de trabalho para poder alcançar o objetivo do estágio ${ }^{27}$.

Quando se evidenciou com os resultados do estudo a importância do investimento na formação docente, foi possível a criação de um desenho de curso on-line para os supervisores de estágio participantes do estudo, a partir do mapeando de suas necessidades, com subsequente aplicação do curso, por meio de ferramentas interativas com ênfase no exercício de comunicação e interação entre os participantes, e uso de metodologias ativas na modalidade on-line ${ }^{38}$.

\section{CONCLUSÃO}

O estudo trouxe à tona a importância da formação pedagógica do preceptor para receber o estudante no local de trabalho e assim exercer sua função de educador das práticas no serviço.

Quanto aos conteúdos pedagógicos necessários ao ensino nos estágios, apontaram-se dois aspectos imprescindíveis: fluxo contínuo de formação e treinamento para a docência no serviço, em especial sobre avaliação de desempenho e práticas inovadoras.

Percebe-se também que a compreensão das necessidades pedagógicas da supervisão de estágio é uma excelente ferramenta para aperfeiçoar o projeto políticopedagógico do curso, bem como para estruturar um desenho de curso de capacitação docente que, no caso, foi realizado na modalidade on-line.

Os discentes disseram que esperam encontrar supervisores que tragam contribuições significativas em sua aprendizagem de uma forma segura, por meio da experiência profissional, demonstrando incentivo e pontuando qualidades e falhas observadas.

O estudo possibilitou ainda compreender que a presença do estudante no serviço contribui para as práticas e a troca de saberes no cotidiano do trabalho. Deve-se destacar que é necessário um maior reconhecimento da atuação do supervisor, pois às demandas do ensino somam-se as demandas do serviço.

Diante da subcategoria "diálogo entre ensino e serviço", foi abordada pelos preceptores a necessidade de reduzir o distanciamento entre os preceptores do serviço e a IES, em que foi relatada a importância de uma maior aproximação do ensino com os serviços. Um outro aspecto importante encontrado foi a necessidade de uma maior aproximação entre teoria e prática e um diálogo qualificado entre ensino e serviço.

Por fim, vale ressaltar que o presente estudo não preenche todas as lacunas necessárias ao entendimento da temática proposta, sendo limitado a uma área do conhecimento, sugerindo, dessa maneira, a ampliação da discussão por meio de outros projetos mais abrangentes.

\section{CONTRIBUIÇÃO DAS AUTORAS}

Adriana Reis de Barros participou da concepção do estudo, da coleta, análise e interpretação dos dados, e da elaboração e revisão do manuscrito. Rozangela Maria de Almeida Fernandes Wyszomirska e Kerle Dayana Tavares de Lucena participaram significativamente da concepção do estudo e da elaboração e revisão do manuscrito. As autoras Adriana Reis de Barros e Rozangela Maria de Almeida Fernandes Wyszomirska foram responsáveis pela exatidão e integridade de todos os aspectos do estudo e da pesquisa. Todas as autoras participaram da aprovação da versão final do manuscrito para publicação.

\section{CONFLITO DE INTERESSES}

Declaramos não haver conflito de interesses.

\section{FINANCIAMENTO}

Declaramos não haver financiamento.

\section{REFERÊNCIAS}

1. Secretaria de Saúde do Rio de Janeiro. Bases para diálogos e reflexões em educação permanente em saúde. 2a ed. Rio de Janeiro: Secretaria de Saúde do Rio de Janeiro; 2019.

2. Ribeiro PKC, Firmo WDA, Sousa MHSL, Figueiredo IA, Pacheco MAB. Os profissionais de saúde e a prática de preceptoria na atenção básica: assistência, formação e transformações possíveis. J Manag Prim Health Care. 2020;12(1):1-18.

3. Brasil. Lei no 11.788 , de 25 de setembro de 2008. Dispõe sobre o estágio de estudantes e dá outras providências [acesso em 20 maio 2020]. Disponível em: http://www.planalto.gov.br/ccivil_03/_ato2007-2010/2008/lei/111788.htm.

4. Colliselli L, Tombini LHT, Leba ME, Reibnitz KS. Estágio curricular supervisionado: diversificando cenários e fortalecendo a interação ensino-serviço. Rev Bras Enferm. 2009;62(6):932-7.

5. Benito GAV, Tristão KM, Paula ACSF. Desenvolvimento de competências gerais durante o estágio supervisionado. Rev Bras Enferm. 2012;65(1):172-8.

6. Lima TC, Paixão RFC, Cândido EC, Campos CJG, Ceolim MF. Estágio curricular supervisionado: análise da experiência discente. Rev Bras Enferm. 2014;67(1):133-40.

7. Santos EF, Souza FB, Dantas MMCM, Jamelli SR, Carvalho EJA. Estágios curriculares de odontologia nos serviços públicos de saúde após as Diretrizes Curriculares Nacionais de 2002. Rev ABENO. 2018;18(4):31-9.

8. Pimentel EC, Vasconcelos MVL, Rodarte RS, Pedrosa CMS, Pimentel FSC. Ensino e aprendizagem em estágio supervisionado: estágio integrado em saúde. Rev Bras Educ Med. 2015;39(3):352-8.

9. Braga LAV, Morais JDM, Alvarenga JPO, Meneses LBA, Soares VL. Redeescola: um diálogo necessário. In: Ceccim RB, et al., organizadores. Formação de formadores para residências em saúde: corpo docente ap4960ssistencial em experiências vivas. Porto Alegre: Rede Unida; 2016:49-60 
10. Marran AL, Lima PG, Bagnato MHS. As políticas educacionais e o estágio curricular supervisionado no curso de graduação em enfermagem. Trab Educ Saúde. 2015;13(1):89-108.

11. Esteves LSF, Cunha ICKO, Bohomol E, Negri EC. O estágio curricular supervisionado na graduação em enfermagem: revisão integrativa. Rev Bras Enferm. 2018;71(4):1740-50.

12. Lacerda $L C A$, Teles RBA, Omena CMB. Estágio supervisionado: percepção do preceptor sobre o processo de ensino-aprendizagem em um hospital de ensino. Rev e-Curriculum. 2019;17(2): .574-91.

13. Feuerwerker LCM. As identidades do preceptor: assistência, ensino, orientação. In: Ribeiro VMB, organizadora. Formação pedagógica de preceptores do ensino em saúde. Juiz de Fora: Editora UFJF; 2011.126

14. Rodrigues AMM, Freitas CHA, Guerreiro MGS, Jorge MSB. Preceptoria na perspectiva da integralidade conversando com enfermeiros. Rev Gaucha Enferm. 2014;35(2):106-12.

15. Félix TA, Oliveira EN, Araújo MSD. Preceptoria em serviço na rede de urgência e emergência: trilhando novos caminhos para a formação em saúde. In: Ceccim RB, et al., organizadores. Práticas compartilhadas e exigências de interação: envolvimento e trocas em equipe na saúde. Porto Alegre: Rede Unida; 2020: 87-97.

16. Cerqueira P. A formação pedagógica de preceptores dos estudantes da área da saúde: uma conversa em três tempos. In: Ribeiro VMB, organizadora. Formação pedagógica de preceptores do ensino em saúde. Juiz de Fora: Editora UFJF; 2011. p. 67-76.

17. Martins JJN. Formação de preceptores para área de saúde. In: Ribeiro VMB, organizadora. Formação pedagógica de preceptores do ensino em saúde. Juiz de Fora: Editora UFJF; 2011. p. 47-52.

18. Oliveira FD. A preceptoria na estratégia saúde da família: o olhar dos profissionais de saúde. [dissertação]. Natal: Universidade Federal do Rio Grande do Norte; 2014.

19. Meneses LBA, Leite VS, Pereira AJ, Rocha RCS, Fernandes MCV. Vivendo a formação de preceptores e tutores: uma experiência refletida. In: Ceccim $\mathrm{RB}$, et al., organizadores. Formação de formadores para residências em saúde: corpo docente assistencial em experiências viva. Porto Alegre: Rede Unida; 2018:.61-74.

20. Soares $A C P$, Maiorquim CR, Souza CRO, Vale DNF, Fujimoto DE, Fagundes $\mathrm{FP}$, et al. A importância da regulamentação da preceptoria para a melhoria da qualidade dos programas de residência médica na Amazônia Ocidental. Cad ABEM. 2013;9(1):14-23.

21. Autonomo FROM, Hortale VA, Santos GB, Botti SHO. A preceptoria na formação médica e multiprofissional com ênfase na atenção primária análise das publicações brasileiras. Rev Bras Educ Med. 2015;39(2):316-27.

22. Silva EMM, Tourinho FSV, Girondi JBR, Sebold LF, Diniz J, Barbosa JG, et al. Curso EAD de preceptoria em ensino na saúde: uma experiência exitosa. J Bras Telessaúde. 2016;4(2):261-7.
23. Mohr AA. Formação pedagógica dos profisisonais da área da saúde. In: Ribeiro VMB, organizadora. Formação pedagógica dos preceptores do ensino em saúde. Juiz de Fora: Editora UFJF; 2011:574-91.

24. Lima PAB, Rozendo CA. Desafios e possibilidades no exercício da preceptoria do Pró-Pet-Saúde. Interface Comun Saúde Educ. 2015;19(1):779-91.

25. Oliveira MS, Petta HL, Ribeiro ECO. O curso de especialização em preceptoria no SUS 2017. In: Oliveira MS et al. Preceptoria no SUS: caderno do curso. São Paulo: Hospital Sírio-Libanês, Ministério da Saúde; 2017.

26. Ta ACALL, Santana MNS, Pinto ESG, Fernandes MCS, Fernandes VFS. Repensando a trajetória do curso de especialização em preceptoria residência médica e preceptoria no SUS e seus impactos na vida profissional/na vida acadêmica. Cid Ação. 2019;4(2):86-103.

27. Girotto LC. Preceptores do Sistema Único de Saúde: como percebem seu papel em processos educacionais na saúde [dissertação]. São Paulo: Universidade de São Paulo; 2016.

28. Correa GT, Carbone TRJ, Rosa MFAP, Marinho GD, Ribeiro VMB, Motta JIJ. Uma análise crítica do discurso de preceptores em processo de formação pedagógica. Pro-Posições. 2015;26(3):167-78.

29. Bardin L. Análise de conteúdo. São Paulo: Edições 70; 2016.

30. Ferreira FC, Dantas FC, Valente GSC. Saberes e competências do enfermeiro para preceptoria em unidade básica de saúde. Rev Bras Enferm. 2018;71(4):1564-71.

31. Ribeiro KRB, Prado ML. A prática educativa dos preceptores nas residências em saúde: um estudo de reflexão. Rev Gaucha Enferm. 2014;35(1):161-5.

32. Rocha PF, Warmling CM, Toassi RFC. Preceptoria como modalidade de ensino na saúde: atuação e características do preceptor cirurgião-dentista da atenção primária. Rev Saberes Plurais. 2016;1(1):96-112.

33. Gubert $E$, Prado ML. Desafios na prática pedagógica na educação profissional em enfermagem. Rev Eletr Enferm. 2011;13(2):285-95.

34. Missaka $H$, Ribeiro VMB. A preceptoria na formação médica: o que dizem os trabalhos nos congressos brasileiros de educação médica 2007-2009. Rev Bras Educ Med. 2011;35(3):303-10.

35. Teixeira RC, Domingues RJS, Kietzer KS, Araújo RMS, Gonçalves KLP. Preceptoria em saúde: definição do papel do preceptor. Cad Educ Saúde Fisiot. 2018;5(10). 578-83

36. Ferreira AMM. Percepção dos discentes de nutrição sobre a formação para a atenção básica. [dissertação]. Maceió: Universidade Estadual de Ciências da Saúde de Alagoas; 2019.

37. Junqueira SR, Oliver FC. A preceptoria em saúde em diferentes cenários de prática. Rev Docência Ensino Super. 2020;10(1):1-20.

38. Barros AR, Wyszomirska RMAF, Lucena KDT. Open educational product: online course design for internship supervisors of an undergraduate course in the health area. Creative Education. 2021;12:1397-406. 